[Article]

www.whxb.pku.edu.cn

\title{
羟基加成反应对 A-T碱基对结构和质子转移过程的影响
}

\author{
史俊友 ${ }^{1,2, *}$ 董丽花 ${ }^{1,2}$ 刘永军 ${ }^{1}$ \\ ('中国科学院西北高原生物研究所, 西宁 810001; 2中国科学院研究生院, 北京 100049)
}

\begin{abstract}
摘要：应用密度泛函理论在 B3LYP/6-31++G $(d, p) / / \mathrm{B} 3 \mathrm{LYP} / 6-31 \mathrm{G}(d, p)$ 水平上对羟基化碱基对 A-T 的结构进 行了研究, 经计算共得到 8 种稳定的羟基化加成产物, 其能量的相对顺序为 ${ }^{8 \mathrm{OH}} \mathrm{A}-\mathrm{T}<\mathrm{A}-\mathrm{T}^{6 \mathrm{OH}}<\mathrm{A}-\mathrm{T}^{5 \mathrm{OH}}<{ }^{20 \mathrm{H}} \mathrm{A}-\mathrm{T}<$ ${ }^{40 \mathrm{H}} \mathrm{A}-\mathrm{T}<{ }^{5 \mathrm{OH}} \mathrm{A}-\mathrm{T}<\mathrm{A}-\mathrm{T}^{20 \mathrm{H}}<\mathrm{A}-\mathrm{T}^{4 \mathrm{OH}}$ (数字表示羟基加成进攻的原子在腺嘌呤或胸腺嘧啶中的编号), 这与其加成反应 前后结构变化的大小密切相关. 当差基对腺嘌呤端进行加成时, A-T 间的相互作用能略有增加, 而当羟基对胸 腺嘧啶进行加成时, A-T之间的相互作用能略有减小. 另外, 还以能量较低的加成产物 ${ }^{80 \mathrm{H}} \mathrm{A}-\mathrm{T}$ 和 A-T $\mathrm{T}^{6 \mathrm{OH}}$ 为例对羟 基化碱基对中 $\mathrm{A}$ 和 $\mathrm{T}$ 之间的质子转移过程进行了研究, 结果表明羟基化产物中 $\mathrm{A}$ 与 $\mathrm{T}$ 之间的质子转移机理由未 加成前的分步双质子转移变为协同双质子转移, 且其势垒低于未加成的 A-T 发生第一步(决速步骤)质子转移的 势垒.
\end{abstract}

关键词: 密度泛函理论; 碱基对; 羟基化; 质子转移; 单电子占据轨道 中图分类号: 0641

\section{Effect of Hydroxylation on Structures and Proton Transfer of A-T Base Pairs}

\author{
SHI Jun-You ${ }^{1,2, *} \quad$ DONG Li-Hua ${ }^{1,2} \quad$ LIU Yong-Jun ${ }^{1}$ \\ ('Northwest Institute of Plateau Biology, Chinese Academy of Sciences, Xining 810001, P. R. China; \\ ${ }^{2}$ Graduate University of Chinese Academy of Sciences, Beijing 100049, P. R. China)
}

\begin{abstract}
The structures and proton transfer processes of hydroxylated A-T base pairs were theoretically studied at the B3LYP/6-31++ $(d, p) / / \mathrm{B} 3 \mathrm{LYP} / 6-31 \mathrm{G}(d, p)$ level. Our calculations revealed that hydroxyl radical could react with $A-T$ at different positions to form eight stable adducts. The order of these adducts in energy is ${ }^{80 \mathrm{H}} \mathrm{A}-\mathrm{T}<\mathrm{A}-\mathrm{T}^{60 \mathrm{H}}<\mathrm{A}-\mathrm{T}^{5 \mathrm{OH}}<{ }^{20 \mathrm{H}} \mathrm{A}-\mathrm{T}<{ }^{40 \mathrm{H}} \mathrm{A}-\mathrm{T}<{ }^{50 \mathrm{H}} \mathrm{A}-\mathrm{T}<\mathrm{A}-\mathrm{T}^{20 \mathrm{H}}<\mathrm{A}-\mathrm{T}^{40 \mathrm{H}}$ (the number denotes the label of the atom in the $A / T$ which is attacked by hydroxyl), which relates well with their structural changes upon the addition of hydroxyl radical. The interaction energy between $A$ and $T$ would increase slightly when hydroxyl radical reacts with the adenine, but it would decrease when the radical reacts with thymine. To study the proton transfer processes of the hydroxylated A-T base pairs, the most stable adducts, ${ }^{8 \mathrm{OH}} \mathrm{A}-\mathrm{T}$ and $A-T^{60 H}$, were selected to give calculations. The calculated results indicate that the proton transfer processes of ${ }^{80 \mathrm{H}} \mathrm{A}-\mathrm{T}$ and $\mathrm{A}-\mathrm{T}^{6 \mathrm{OOH}}$ follow the concerted mechanism, which is different from the stepwise mechanism of A-T. What is more, its energy barrier is lower than the corresponding energy of the latter's first step (rate-determining step).
\end{abstract}

Key Words: Density functional theory; Base pair; Hydroxylation; Proton transfer; Singly occupied molecular orbital

Received: August 2, 2010; Revised: September 13, 2010; Published on Web: October 18, 2010.

"Corresponding author. Email: shijunyou123@126.com; Tel: +86-15809781546.

The project was supported by the Program of "One Hundred Talents Program” of the Chinese Academy of Sciences.

中国科学院“百人计划”研究项目资助

(C) Editorial office of Acta Physico-Chimica Sinica 
在 DNA 的双螺旋结构中, 各个碱基之间通过氢 键相互作用以 A-T(腺嘌呤-胸腺嘧啶)或 G-C(岛嘌 呤-胞嘧啶)的方式进行配对. 由于受环境因素的影 响, 这些碱基对之间的结合能力可能会发生变化, 甚至诱发突变. 生物体系内的水分子经紫外线辐射 或细胞的正常代谢后可分解产生活性基团如 $\mathrm{H} \cdot$ 、 $\cdot \mathrm{OH} 、 \mathrm{O}_{2}^{-} 、 \mathrm{H}_{2} \mathrm{O}_{2}$ 等. 这些活性基团可与核苷作用导致 DNA 发生一系列的变化, 如单、双螺旋链结构的变 化, 碱基对间的错配, 突变等. 由于这些自由基与核 苷的反应具有严重的破坏性, 其反应机理已经受到 人们的密切关注.

人们通过实验及理论研究发现 $\cdot \mathrm{OH}$ 与 DNA 中 的嘧啶(胸腺嘧啶或胞嘧啶)及嘌呤(腺嘌呤或岛嘌 呤)发生加成反应, 且其相关产物已得到分离和鉴 定 $^{[1-2]} \cdot \cdot \mathrm{OH}$ 与嘧啶的加成反应主要发生在 C5 及 $\mathrm{C} 6$ 位, 其中 C5 位的加成产物具有还原性, C6 位的加成 产物具有氧化性; $\cdot \mathrm{OH}$ 与嘌呤的加成反应可发生在 C2、C4、C5、C8 等位置, 其产物中氧化性和还原性物 质呈等量关系 ${ }^{[3-5]}$. 这些加成产物仍比较活泼, 不但 可与其它生物分子发生反应, 还可发生分子内的开 环反应、分子内及分子间的抽氢反应等 ${ }^{[5-6]}$. 通常情 况下, 核酸中的嘌呤和嘧啶主要以氨基和酮类的形 式通过分子间氢键进行配对, 但在少数情况下, 嘌 呤和嘧啶也以亚氨基和烯醇的形式存在(图 1). 后一 种情况的发生是由于嘌呤与嘧啶之间发生了质子 转移 ${ }^{[7-9]}$; 它所占比例虽然很小, 却可以诱发基因突
变, 改变遗传密码 ${ }^{[10]}$.

为探讨碱基对之间的质子转移及其在基因突 变中的作用, 人们开展了许多相关的实验及理论研 究. 结果表明 G-C 间的质子转移遵循协同双质子转 移机理 ${ }^{[1]}$, 但对于碱基对 A-T 间的质子转移机理, 人 们得到的结论并不完全一致, 如 Floribn ${ }^{[12]}$ 和 Gorb ${ }^{[13]}$ 等认为碱基对 A-T 间的质子转移机理为协同双质子 转移, 而近期 Kryachk 等 ${ }^{[14]}$ 和 Villani ${ }^{[15]}$ 的研究发现 A-T 间的质子转移为分步质子转移. 在分步质子转 移中, 首先胸腺嘧啶的 $\mathrm{H} 8$ 由 $\mathrm{N} 3$ 转移到腺嘌呤的 $\mathrm{N} 1$ 上, 形成离子型中间体 $\mathrm{A}-\mathrm{T}^{\prime}$, 然后腺嘌呤 $\mathrm{N} 10$ 上的 氢原子转移到胸腺嘧啶的 $\mathrm{O} 9$ 上形成 $\mathrm{AT}^{\prime} \mathrm{T}^{\prime}$ 图 1); Kryachk 等 ${ }^{[14]}$ 在 $\mathrm{HF} / 6-31+\mathrm{g}(d)$ 水平上计算得到的分 步质子转移的能垒分别为 70.26 和 $37.22 \mathrm{~kJ} \cdot \mathrm{mol}^{-1}$.

活性自由基与碱基相互作用的研究引起了人 们的极大关注 ${ }^{[1-6,16-19]}$, 但由于这些自由基活性较高, 采用实验方法对其研究具有较大的局限性, 于是人 们采用多种理论方法对着基与单个碱基的相互作 用进行了较为深入的研究, 但对于羟基与碱基对之 间的相互作用及其对碱基对之间质子转移过程影 响的研究还相对较少. Zhang 等 ${ }^{[20]}$ 用密度泛函方法 研究了羟基自由基与 $\mathrm{G}-\mathrm{C}$ 碱基对的加成反应及碱 基之间的质子转移过程, 他们发现羟基加成对于 $\mathrm{G}$ 与 $\mathrm{C}$ 之间的相互作用有轻微的影响, 但加成位置对 $\mathrm{G}$ 与 $\mathrm{C}$ 之间的质子转移势垒有不同的影响. 到目前 为止, 对于差基自由基与碱基对 A-T之间的加成反
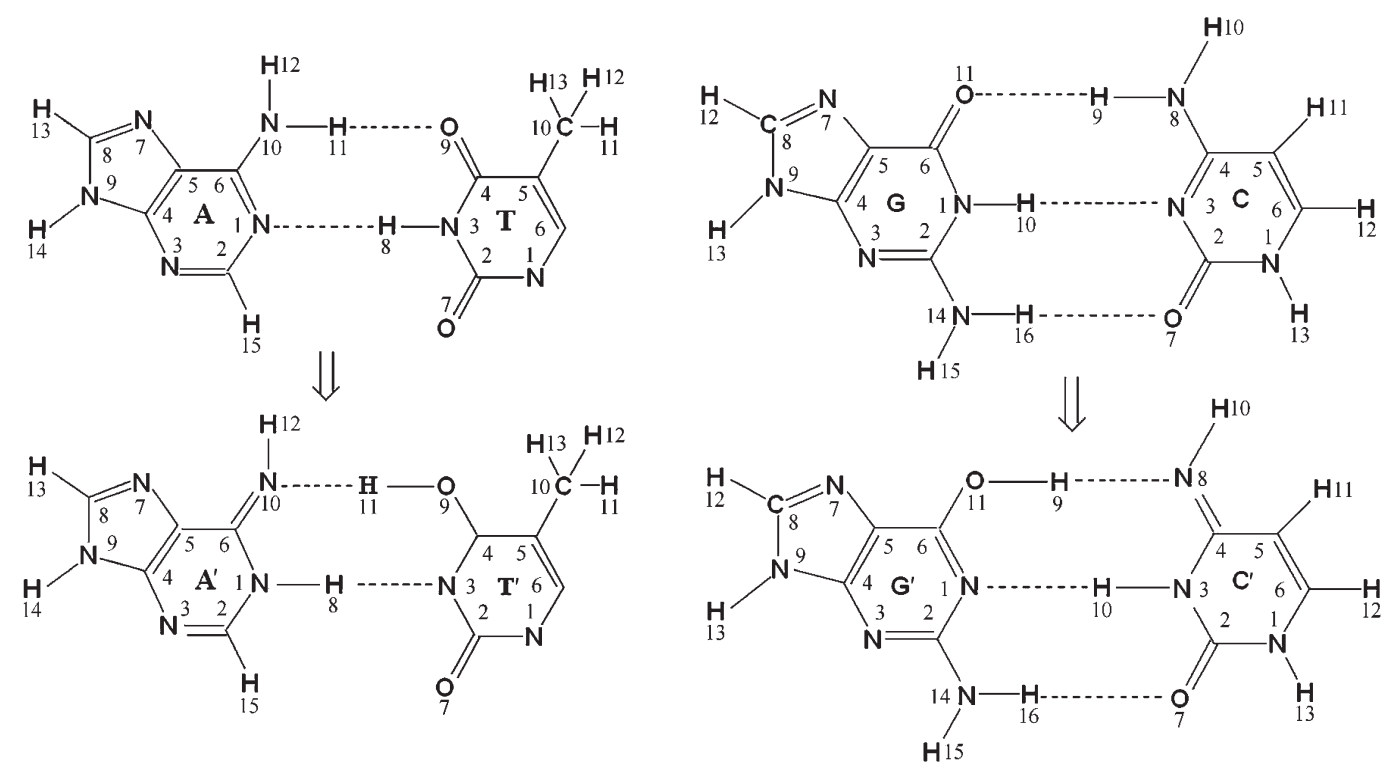

图 1 碱基对 A-T 和 G-C 及其质子转移产物的原子编号标注示意图

Fig.1 Atom numbering scheme of A-T and G-C base pairs and their proton transfer products 
应尚未见文献报道. 因此本文用密度泛函理论 ${ }^{[21-22]}$ 对羟基加成后碱基对的结构变化以及 A- $\mathrm{T}$ 间的质子 转移过程进行了研究, 以期加深对 DNA 损伤和修复 过程的理解.

\section{1 计算方法}

因本文研究体系涉及到较多的氢键相互作用, 我们采用已被证明对氢键体系十分有效的 B 3 LYP 方法 ${ }^{[23-24]}$ 在 6-31G $(d, p)$ 水平上对可能的羟基化产物 及其质子转移过程涉及到的各驻点的构型进行了 全优化, 并通过频率计算加以确认; 所有的局域最 小点没有虚频, 而相应的过渡态只有一个虚频; 过 渡态还通过内毫反应坐标(IRC)加以确认, 自然布居 通过自然键轨道分析(NBO)得到. 为使计算过程中 所涉及的复合物、过渡态及产物的能量更加准确, 在 B3LYP/6-31++ G $(d, p)$ 水平上对各优化构型进行 了单点计算, 下文中提到的能量都是指 B3LYP/ $6-31++\mathrm{G}(d, p)$ 单点计算后所得到的能量(包含零点能 校正). 全部计算采用 Gaussian 03 程序包 ${ }^{[25]}$ 完成.

为从本质上阐述 $\mathrm{A}-\mathrm{T}$ 碱基对之间质子转移势垒 变化的原因, 本文还计算了有关羟基化碱基的质子 亲和势 $(\mathrm{PA})$ 与去质子焓 $(\mathrm{DPE})$.

对于质子化过程: $\mathrm{B}+\mathrm{H}^{+} \rightarrow \mathrm{BH}^{+}$

反应的吉布斯自由能变化通过下式计算:

$$
\begin{aligned}
\Delta H & =E\left(\mathrm{BH}^{+}\right)-E(\mathrm{~B})-E\left(\mathrm{H}^{+}\right)+\Delta p V \\
& =E\left(\mathrm{BH}^{+}\right)-E(\mathrm{~B})+E_{\text {vib }}\left(\mathrm{BH}^{+}\right)-E_{\text {vib }}(\mathrm{B})-R T \\
\Delta G & =\Delta H-T \Delta S \\
\Delta S & =S\left(\mathrm{BH}^{+}\right)-S(\mathrm{~B})-S\left(\mathrm{H}^{+}\right)
\end{aligned}
$$

其中 $E(\mathrm{i}) 、 E_{\mathrm{vib}}(\mathrm{i})$ 和 $S(\mathrm{i})$ 分别代表各物种 $\mathrm{i}$ 的总能量、 零点振动能及熵; $T$ 和 $R$ 分别为物质的绝对温度及 气体常数; 质子亲和势 PA 为吉布斯自由能变化
$(\Delta G)$ 的负值. 对于质子化过程来说, PA 的值越大, 则 该碱的碱性越强.

对于去质子化过程: $\mathrm{BH} \rightarrow \mathrm{B}^{-}+\mathrm{H}^{+}$

其焓变计算公式与式(1)相似:

$$
\begin{aligned}
\Delta H & =E\left(\mathrm{~B}^{-}\right)+E\left(\mathrm{H}^{+}\right)-E(\mathrm{BH})+\Delta p V \\
& =E\left(\mathrm{~B}^{-}\right)-E(\mathrm{BH})+E_{\mathrm{vib}}\left(\mathrm{B}^{-}\right)-E_{\mathrm{vib}}(\mathrm{BH})+R T
\end{aligned}
$$

去质子焓 DPE 为该过程函变 $(\Delta H)$ 的负值. 一般情况 下, 去质子焓越小表明该物质 $(\mathrm{BH})$ 的酸性越强.

本文中所涉及的原子编号始终与图 1 中 A-T 的 编号一致. 依据羟基加成位置的不同本文将 $\cdot \mathrm{OH}$ 与 $\mathrm{A}-\mathrm{T}$ 的加成产物命名为 ${ }^{\mathrm{MOH}} \mathrm{A}-\mathrm{T}$ 或 A-T ${ }^{\mathrm{NOH}}$, 前者代表 着基加成到腺嘌呤上, 后者代表羟基加成到胸腺嘧 啶上, $M$ 和 $N$ 分别代表羟基加成进攻的原子在腺嘌 呤和胸腺嘧啶中的编号, ${ }^{\mathrm{MOH}} \mathrm{A}-\mathrm{T}(\mathrm{Ts} / \mathrm{P})$ 和 $\mathrm{A}-\mathrm{T}^{\mathrm{NOH}}(\mathrm{Ts} /$ P)分别代表质子转移过程的过渡态或转移后的产 物.

\section{2 结果与讨论}

\section{1 加成产物的结构}

对 A-T 碱基对中所有可能与 $\cdot \mathrm{OH}$ 发生加成反应 的不饱和原子进行了尝试, 包括腺嘌呤 $(\mathrm{A})$ 中的 2-8 位原子, 胸腺嘧啶 $(\mathrm{T})$ 中的 2-6位原子. 经计算得到 了 8 种稳定的加成产物, 分别是 ${ }^{2 \mathrm{OH}} \mathrm{A}-\mathrm{T} 、{ }^{4 \mathrm{OH}} \mathrm{A}-\mathrm{T}$ 、 ${ }^{5 \mathrm{OH}} \mathrm{A}-\mathrm{T} 、{ }^{8 \mathrm{OH}} \mathrm{A}-\mathrm{T} 、 \mathrm{~A}-\mathrm{T}^{2 \mathrm{OH}} 、 \mathrm{~A}-\mathrm{T}^{4 \mathrm{OH}} 、 \mathrm{~A}-\mathrm{T}^{5 \mathrm{OH}}$ 和 $\mathrm{A}-\mathrm{T}^{6 \mathrm{OH}}$.

表 1 中列出了羟基氧原子与被加成原子所形成 键的键长及相关原子上的电子自旋密度. 可以看 出, 羟基氧原子上的电子自旋密度都很小, 表明其 单电子已经转移到被加成碱基上; 羟基与被加成原

\begin{tabular}{|c|c|c|c|c|c|}
\hline System & $\rho(\mathrm{i})^{\mathrm{b}}$ & $\rho(\mathrm{i})^{\mathrm{b}}$ & $\rho(\mathrm{i})^{\mathrm{b}}$ & $\rho(\mathrm{O})^{\mathrm{c}}$ & $R_{\mathrm{X}-\mathrm{OH}}{ }^{\mathrm{d}} / \mathrm{nm}$ \\
\hline${ }^{2 \mathrm{OH}} \mathrm{A}-\mathrm{T}^{\mathrm{a}}$ & $0.4759(\mathrm{C} 2)$ & 0.5857 (C6) & $0.5269(\mathrm{C} 4)$ & -0.1943 & 0.1422 \\
\hline${ }^{4 \mathrm{H}} \mathrm{A}-\mathrm{T}$ & $-0.0156(\mathrm{C} 4)$ & 0.5041 (C5) & $0.2556(\mathrm{C} 8)$ & 0.0358 & 0.1420 \\
\hline${ }^{5 \mathrm{OH}} \mathrm{A}-\mathrm{T}$ & $-0.0030(\mathrm{C} 5)$ & $0.3950(\mathrm{C} 2)$ & $0.3612(\mathrm{C} 4)$ & 0.0481 & 0.1442 \\
\hline${ }^{8 \mathrm{OH}} \mathrm{A}-\mathrm{T}$ & 0.0190 (C8) & 0.4644 (N7) & $0.1819(\mathrm{C} 2)$ & 0.0245 & 0.1400 \\
\hline $\mathrm{A}-\mathrm{T}^{2 \mathrm{OH}}$ & $-0.0480(\mathrm{C} 2)$ & $0.5696(\mathrm{O} 7)$ & $0.2915(\mathrm{~N} 1)$ & 0.0083 & 0.1398 \\
\hline $\mathrm{A}-\mathrm{T}^{4 \mathrm{OH}}$ & $-0.0530(\mathrm{C} 4)$ & $0.2082(\mathrm{~N} 1)$ & $0.7828(\mathrm{O} 9)$ & 0.0246 & 0.1404 \\
\hline $\mathrm{A}-\mathrm{T}^{5 \mathrm{OH}}$ & $-0.0360(\mathrm{C} 5)$ & 0.7947 (C6) & & 0.0664 & 0.1445 \\
\hline $\mathrm{A}-\mathrm{T}^{6 \mathrm{OH}}$ & $-0.0437(\mathrm{C} 6)$ & $0.8383(\mathrm{C} 5)$ & & 0.0490 & 0.1415 \\
\hline
\end{tabular}
子形成了稳定的化学键, 键长在 0.1398-0.1445 nm 之间。

表 2 列出了 8 种羟基化产物的总能量和相对能

表 1 羟基化碱基对中相关原子的电子自旋密度及 $\mathbf{X}$ - OH 的键长

Table 1 Electron spin density of related atoms and bond length of $\mathrm{X}-\mathrm{OH}$ for hydroxylated base pairs

${ }^{\text {a }{ }^{\circ} \mathrm{H}} \mathrm{A}-\mathrm{T} / \mathrm{A}-\mathrm{T}^{\mathrm{NOH}}$ represents the A-T base pair reacting with the hydroxyl on $\mathrm{A} / \mathrm{T}$ 's $M$ th $/ N$ th atom. ${ }^{\mathrm{b}} \mathrm{Only}$ the spin densities with large value and those for addition atom which reacted with the hydroxyl are listed, the numbers in parentheses represent the related atoms of the base. ${ }^{\mathrm{c}} \rho(\mathrm{O})$ presents the spin densities of oxygen atom of hydroxyl. ${ }^{\mathrm{d}} R_{\mathrm{X}-\text { он }}$ denotes bond length. $\mathrm{X}$ denotes the atom where the hydroxyl adds on. 
表 2 羟基化碱基对 $\mathrm{A}-\mathrm{T}$ 的相对能量 $\left(\boldsymbol{E}_{\mathrm{R}}\right)$ 及相应碱基间的 相互作用能 $\left(\Delta E_{\text {inter }}\right)$

Table 2 Relative energies $\left(E_{\mathrm{R}}\right)$ of hydroxylated A-T and interaction energies $\left(\Delta E_{\text {inter }}\right)$ between related bases

\begin{tabular}{ccc}
\hline System & $E_{\mathrm{R}} /\left(\mathrm{kJ} \cdot \mathrm{mol}^{-1}\right)$ & $\Delta E_{\text {incer }}\left(\mathrm{kJ} \cdot \mathrm{mol}^{-1}\right)$ \\
\hline $\mathrm{A}-\mathrm{T}$ & & 46.33 \\
${ }^{8 \mathrm{OH}} \mathrm{A}-\mathrm{T}$ & 0.00 & 48.82 \\
$\mathrm{~A}^{6 \mathrm{OH}}$ & 24.47 & 43.27 \\
$\mathrm{~A}^{5 \mathrm{OH}}$ & 47.57 & 44.47 \\
${ }^{20 \mathrm{H}} \mathrm{A}-\mathrm{T}$ & 55.22 & 47.07 \\
${ }^{40 \mathrm{H}} \mathrm{A}-\mathrm{T}$ & 80.19 & 52.56 \\
${ }^{5 \mathrm{OH}} \mathrm{A}-\mathrm{T}$ & 84.15 & 50.35 \\
$\mathrm{~A}-\mathrm{T}^{2 \mathrm{OH}}$ & 144.42 & 41.96 \\
$\mathrm{~A}-\mathrm{T}^{4 \mathrm{OH}}$ & 153.20 & 35.81 \\
\hline
\end{tabular}

量, 其中 ${ }^{8 \mathrm{OH}} \mathrm{A}-\mathrm{T}$ 的能量最低, $\mathrm{A}-\mathrm{T}^{40 \mathrm{H}}$ 能量最高, 其能 量由低到高的顺序为 ${ }^{8 \mathrm{OH}} \mathrm{A}-\mathrm{T}<\mathrm{A}-\mathrm{T}^{6 \mathrm{OH}}<\mathrm{A}-\mathrm{T}^{5 \mathrm{OH}}<{ }^{2 \mathrm{OH}} \mathrm{A}-\mathrm{T}<$ ${ }^{4 \mathrm{OH}} \mathrm{A}-\mathrm{T}<{ }^{5 \mathrm{OH}} \mathrm{A}-\mathrm{T}<\mathrm{A}-\mathrm{T}^{2 \mathrm{OH}}<\mathrm{A}-\mathrm{T}^{4 \mathrm{OH}}$. 在腺嘌呤端的 4 种加 成产物中, ${ }^{8 \mathrm{OH}} \mathrm{A}-\mathrm{T}$ 的能量最低, ${ }^{2 \mathrm{OH}} \mathrm{A}-\mathrm{T}$ 次之, 而 ${ }^{4 \mathrm{OH}} \mathrm{A}-\mathrm{T}$ 和 ${ }^{\mathrm{O} H} \mathrm{~A}-\mathrm{T}$ 的能量较高. 在胸腺嘧啶端的 4 种产物中, $\mathrm{A}-\mathrm{T}^{6 \mathrm{OH}}$ 的能量最低, $\mathrm{A}-\mathrm{T}^{5 \mathrm{OH}}$ 次之; 而 $\mathrm{A}-\mathrm{T}^{2 \mathrm{OH}}$ 与 $\mathrm{A}-\mathrm{T}^{4 \mathrm{OH}}$ 能量相当高, 它们分别比 ${ }^{80 \mathrm{H}} \mathrm{A}-\mathrm{T}$ 的能量高 144.42 和 $153.20 \mathrm{~kJ} \cdot \mathrm{mol}^{-1}$, 这与在溶液中未得到 $\mathrm{A}-\mathrm{T}^{2 \mathrm{OH}}$ 和 $\mathrm{A}-\mathrm{T}^{4 \mathrm{OH}}$ 的实验结果 ${ }^{[3-5]}$ 相一致. 由于羟基的加成位置 对产物的稳定性有较大的影响, 我们对腺嘌呤和胸 腺嘧啶加成产物的结构分别进行了讨论.

\subsection{1 腺嘌呤端的加成产物}

图 2 列出了 4 种腺嘌呤端加成产物的结构.
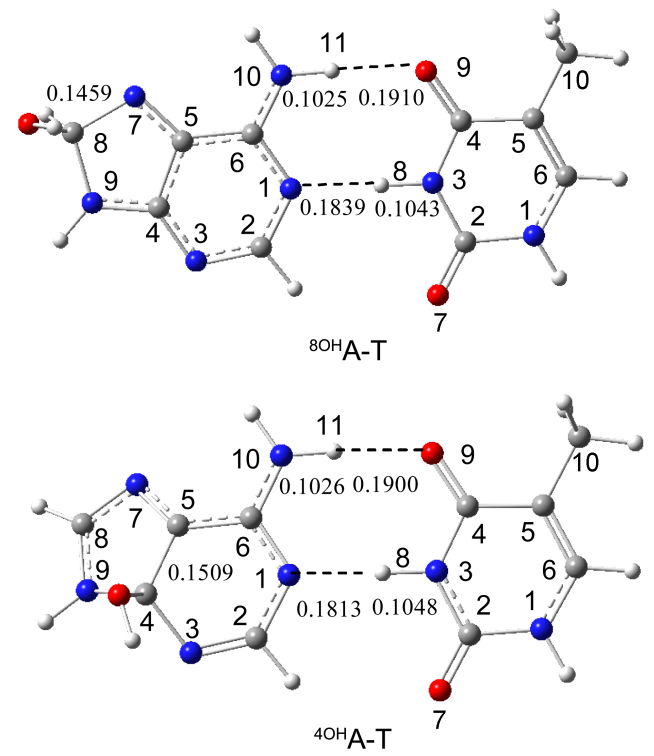

${ }^{80 \mathrm{H}} \mathrm{A}-\mathrm{T}$ 是 $\cdot \mathrm{OH}$ 与腺嘌呤的 $\mathrm{C} 8$ 加成反应的产物, 其能 量在 $\cdot \mathrm{OH}$ 与腺嘌呤端的加成产物中最低. 当 $\cdot \mathrm{OH}$ 进 攻 C8 时, $\mathrm{C} 8$ 的杂化方式由 $s p^{2}$ 转化为 $s p^{3}, \mathrm{C} 8$ 与 $\mathrm{N} 7$ 间的距离由 $0.1310 \mathrm{~nm}$ 增长到 $0.1459 \mathrm{~nm}$, 即 $\mathrm{C} 8$ 与 $\mathrm{N} 7$ 间的化学键由双键变为单键. 在 ${ }^{8 \mathrm{OH}} \mathrm{A}-\mathrm{T}$ 中, 尽管 C8 上的氢原子向嘌呤环外发生扭曲, 但嘌呤环的整 体构型改变很小, 仍基本保持 $C_{s}$ 对称性. ${ }^{20 \mathrm{H}} \mathrm{A}-\mathrm{T}$ 的情 况与 ${ }^{8 \mathrm{OH}} \mathrm{A}-\mathrm{T}$ 类似, 腺嘌呤部分的结构变化不大, 基本 保持 $C_{s}$ 对称性. 而在 ${ }^{4 \mathrm{OH}} \mathrm{A}-\mathrm{T}$ 和 ${ }^{\mathrm{O}}{ }^{\mathrm{OH}} \mathrm{A}-\mathrm{T}$ 中, 由于桥连碳 原子 $\mathrm{C} 4$ 和 $\mathrm{C} 5$ 的杂化方式由 $s p^{2}$ 转化为 $s p^{3}$, 嘌呤环结 构变化较大, 五元环与六元环间呈蝶形扭曲.

羟基与腺嘌呤发生加成后, 碱基对 A-T 间的氢 键键长稍有变化. 在 ${ }^{80 \mathrm{H}} \mathrm{A}-\mathrm{T} 、{ }^{2 \mathrm{OH}} \mathrm{A}-\mathrm{T} 、{ }^{40 \mathrm{H}} \mathrm{A}-\mathrm{T}$ 和 ${ }^{5 \mathrm{OH}} \mathrm{A}-\mathrm{T}$ 中, 氢键 $\mathrm{N} 10-\mathrm{H} 11 \cdots \mathrm{O} 9$ 较 $\mathrm{A}-\mathrm{T}$ (氢键为 $0.1930 \mathrm{~nm}$ ) 中分别缩短了 $0.0020 、-0.0013 、 0.0030$ 和 0.0019 $\mathrm{nm}$, 氢键 $\mathrm{N} 3-\mathrm{H} 8 \cdots \mathrm{N} 1$ 基本未变, 其相应的相互作 用能分别增加了 $2.49 、 0.74 、 6.23$ 和 $4.02 \mathrm{~kJ} \cdot \mathrm{mol}^{-1}$ (表 2). 这说明羟基对腺嘌呤 $\mathrm{C} 8 、 \mathrm{C} 2 、 \mathrm{C} 4$ 和 $\mathrm{C} 5$ 的加 成对 A-T间的氢键有增强作用.

\section{1 .2 胸腺嘧啶端的加成产物}

图 3 为胸腺嘧啶端加成产物的结构. 在 $\mathrm{A}-\mathrm{T}^{5 \mathrm{OH}}$ 的 胸腺嘧啶端, $\mathrm{C} 5$ 的杂化方式由 $s p^{2}$ 转化为 $s p^{3}, \mathrm{C} 5$ 与 C6 间的距离由 $0.1364 \mathrm{~nm}$ 增长到 $0.1489 \mathrm{~nm}$, 二面角 $\mathrm{C} 2-\mathrm{N} 3-\mathrm{C} 4-\mathrm{C} 5$ 与 $\mathrm{C} 2-\mathrm{N} 1-\mathrm{C} 6-\mathrm{C} 5$ 分别由 $0^{\circ}$ 增 加到 $10.67^{\circ}$ 和 $22.63^{\circ}$, 表明 $\mathrm{C} 5$ 已经脱离嘧啶环所在

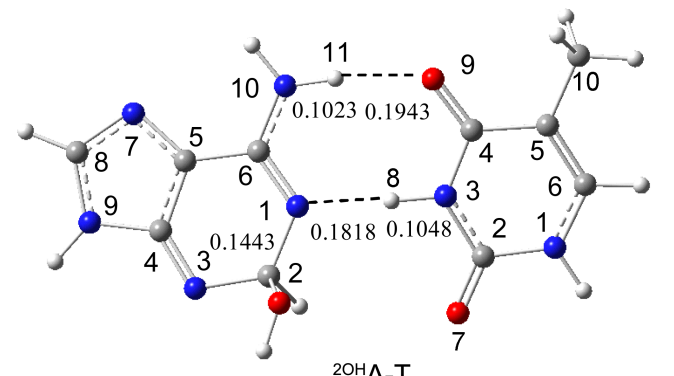

${ }^{2 O H} \mathrm{~A}-\mathrm{T}$

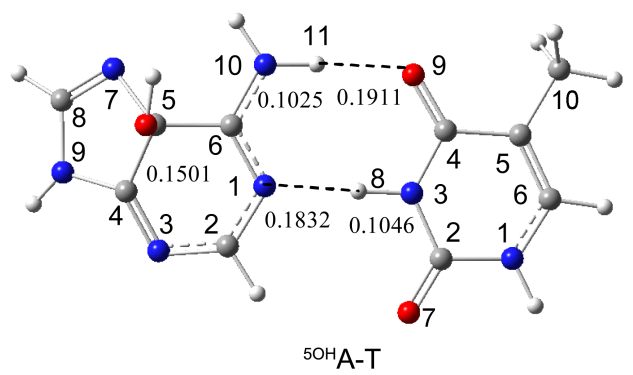

图 $2 \mathrm{~B} 3 \mathrm{LYP} / 6-31 \mathrm{G}(d, p)$ 水平上优化得出的四种 ${ }^{M O H} \mathrm{~A}-\mathrm{T}$ 的相关构型

Fig.2 Four structures of ${ }^{M O H} \mathrm{~A}-\mathrm{T}$ optimized at the B3LYP/6-31G $(d, p)$ level

The unit of bond length in this figure and the following figures is $\mathrm{nm}$. 


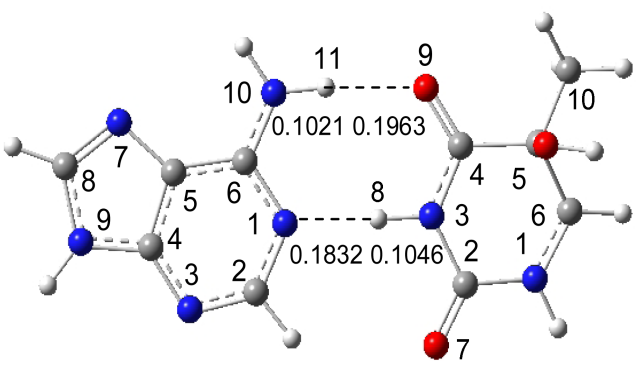

$\mathrm{A}-\mathrm{T}^{5 \mathrm{OH}}$

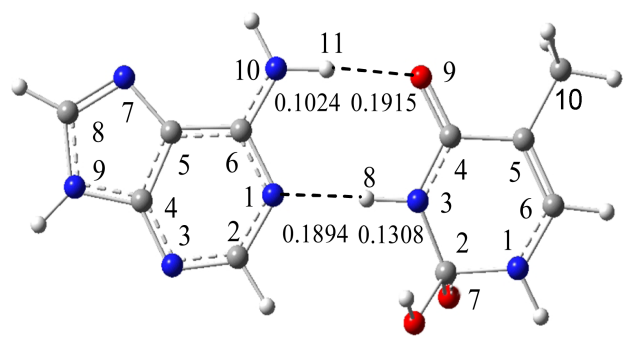

$\mathrm{A}-\mathrm{T}^{2 \mathrm{OH}}$

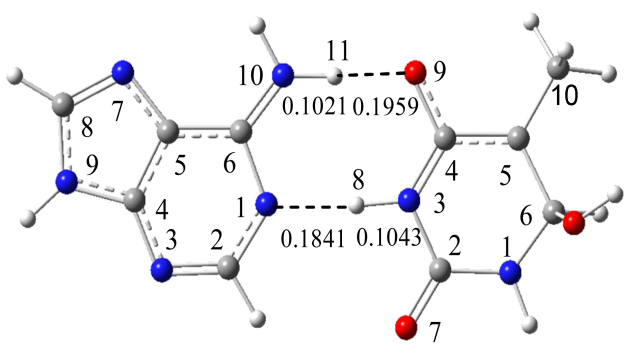

$\mathrm{A}-\mathrm{T}^{6 \mathrm{OH}}$

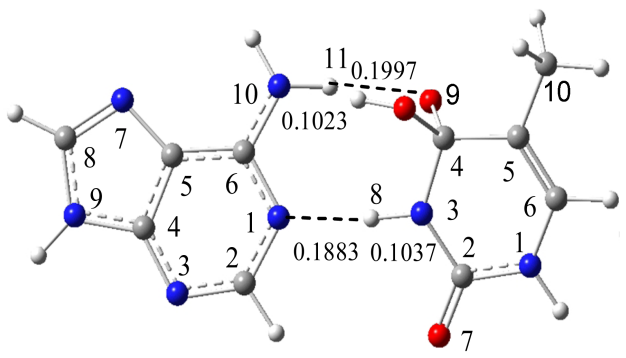

$\mathrm{A}-\mathrm{T}^{4 \mathrm{OH}}$

图 $3 \mathrm{~B} 3 \mathrm{LYP} / 6-31 \mathrm{G}(d, p)$ 水平上优化得出的四种 $\mathbf{A}-\mathrm{T}^{\mathrm{NOH}}$ 的构型

Fig.3 Four structures of A-T ${ }^{\mathrm{NOH}}$ optimized at the B3LYP/6-31G $(d, p)$ level

平面. 而二面角 $\mathrm{N} 1-\mathrm{C} 2-\mathrm{N} 3-\mathrm{C} 4$ 和 $\mathrm{C} 6-\mathrm{N} 1-$ $\mathrm{C} 2-\mathrm{N} 3$ 仅为 $4.32^{\circ}$ 和 $0.51^{\circ}$, 表明胸腺嘧啶环中的其 它五个原子仍基本保持共平面. $\mathrm{A}-\mathrm{T}^{6 \mathrm{OH}}$ 与 $\mathrm{A}-\mathrm{T}^{5 \mathrm{OH}}$ 类 似, C6 脱离原嘧啶环所在平面, 其它五个原子仍基 本保持共平面, 这说明 $\mathrm{A}-\mathrm{T}^{5 \mathrm{OH}}$ 与 $\mathrm{A}-\mathrm{T}^{6 \mathrm{OH}}$ 中的碱基 $\mathrm{T}$ 的结构变化较小. 当羟基与胸腺嘧啶的 $\mathrm{C} 2$ 与 $\mathrm{C} 4$ 加 成时引起碱基 $\mathrm{T}$ 的结构变化较大. 在 $\mathrm{A}-\mathrm{T}^{2 \mathrm{OH}}$ 和 $\mathrm{A}-\mathrm{T}^{4 \mathrm{OH}}$ 中, $\mathrm{C} 2$ 与 $\mathrm{C} 4$ 脱离嘧啶环所在的面, 而 $\mathrm{O} 7$ 与 $\mathrm{O} 9$ 向面 的另一侧扭曲, 其中 $\mathrm{C} 2-\mathrm{O} 7$ 和 $\mathrm{C} 4-\mathrm{O} 9$ 间的距离分 别由 0.1220 和 $0.1235 \mathrm{~nm}$ 增长到 0.1323 和 0.1381 $\mathrm{nm}$. 在 $\mathrm{A}-\mathrm{T}^{4 \mathrm{H}}$ 中, 由于腺嘌呤中氨基与已扭曲的 $\mathrm{O} 9$ 之间的氢键相互作用, 导致嘌呤环和嘧啶环由共面 变为异面, 这可能导致 DNA 链的扭曲.

在 $A-T^{5 \mathrm{OH}} 、 A-\mathrm{T}^{6 \mathrm{OH}} 、 A-\mathrm{T}^{2 \mathrm{OH}}$ 和 $\mathrm{A}-\mathrm{T}^{4 \mathrm{OH}}$ 中, 氢键 $\mathrm{N} 10-$ $\mathrm{H} 11 \cdots \mathrm{O} 9$ 较在 $\mathrm{A}-\mathrm{T}$ 中分别变化了 0.0033 、 $0.0029 、-0.0015$ 和 $0.0067 \mathrm{~nm}$, 氢键 $\mathrm{N} 3-\mathrm{H} 8 \cdots \mathrm{N} 1$ 的 键长分别增长了 $0.0000 、 0.0009 、 0.0062$ 和 0.0051 $\mathrm{nm}$, 相应的相互作用能分别降低了 $1.86 、 3.06 、 4.37$ 、 $10.52 \mathrm{~kJ} \cdot \mathrm{mol}^{-1}$. 这表明羟基对胸腺嘧啶 $\mathrm{C} 5 、 \mathrm{C} 6 、 \mathrm{C} 2$ 和 C4 的加成对 A-T 间的氢键有削弱作用.

通过分析 8 种加成产物的构型与能量发现, 当加 成产物的结构在 A-T 基础上变化较大时, 其能量较 高, 反之能量较低. 当 $\mathrm{A}$ 发生羟基化时, 碱基间的相 互作用增强, 而 $\mathrm{T}$ 发生羟基化时, 相互作用减弱.
2.1.3 A-T 着基加成产物的电子结构

图 4列出了羟基与单个碱基和碱基对加合时产 物的单电子占据轨道 $(\mathrm{SOMO})$ 的能量值. 与各单体 加成产物的单电子占据轨道比较, 碱基对 A-T 加成 产物的单电子占据轨道能量普遍增高. 复合物 ${ }^{40 \mathrm{H}} \mathrm{A}-\mathrm{T} 、{ }^{5 \mathrm{H}} \mathrm{A}-\mathrm{T}$ 及 ${ }^{8 \mathrm{OH}} \mathrm{A}-\mathrm{T}$ 中单电子轨道的能量分别升 高了 $0.003 、 0.006$ 和 0.005 a.u., 而 ${ }^{2 \mathrm{OH}} \mathrm{A}-\mathrm{T}$ 的单电子轨 道能量基本不变. $\mathrm{A}-\mathrm{T}^{2 \mathrm{OH}} 、 \mathrm{~A}-\mathrm{T}^{4 \mathrm{OH}} 、 \mathrm{~A}-\mathrm{T}^{5 \mathrm{OH}}$ 和 $\mathrm{A}-\mathrm{T}^{6 \mathrm{OH}}$ 中 的单电子占据轨道能量分别升高了 $0.032 、 0.010$ 、

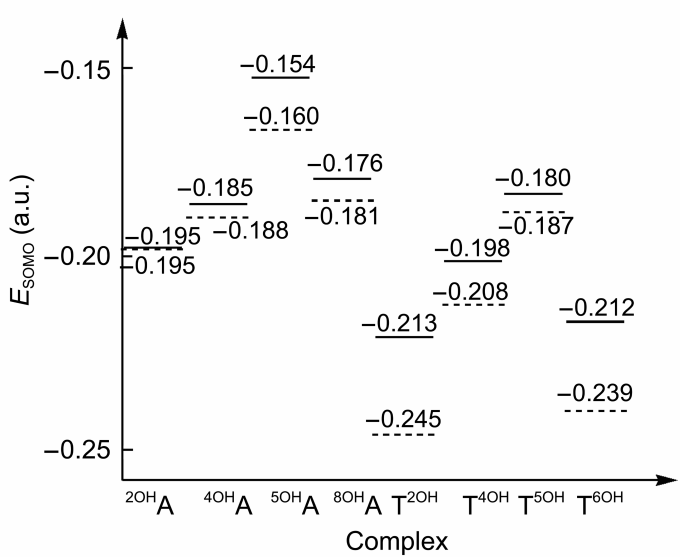

图 4 羟基化碱基单体 $\mathrm{OH}-\mathrm{A} / \mathrm{T}$ (虚线)与相应的羟基化碱基 对 OH-A/T(实线)的单电子占据轨道(SOMO)的能量

Fig.4 Singly occupied molecular orbital (SOMO) energies of monomers $\mathrm{OH}-\mathrm{A} / \mathrm{T}$ (dashed) and corresponding hydroxylation adducts $\mathrm{OH}-\mathrm{A} / \mathrm{T}$ (solid) 

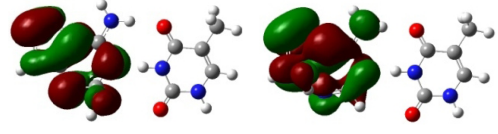

${ }^{2 \mathrm{OH}} \mathrm{A}-\mathrm{T}$

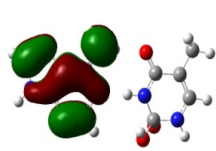

$\mathrm{A}-\mathrm{T}^{2 \mathrm{OH}}$
${ }^{40 \mathrm{OH}} \mathrm{A}-\mathrm{T}$

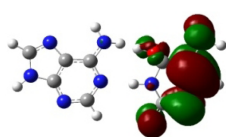

$\mathrm{A}-\mathrm{T}^{4 \mathrm{OH}}$

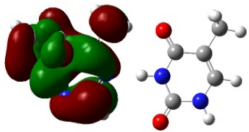

${ }^{5 \mathrm{OH}} \mathrm{A}-\mathrm{T}$

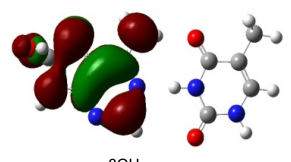

${ }^{80 \mathrm{H}} \mathrm{A}-\mathrm{T}$

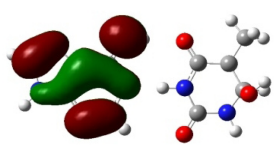

$\mathrm{A}-\mathrm{T}^{6 \mathrm{OH}}$

图 5 复合物 ${ }^{M O H} \mathrm{~A}-\mathrm{T}$ 和 A-T ${ }^{N \mathrm{OH}}$ 的单电子占据轨道图

Fig.5 SOMO populations of adducts ${ }^{M O H} \mathrm{~A}-\mathrm{T}$ and $\mathrm{A}-\mathrm{T}^{\mathrm{NOH}}$

0.007 和 0.027 a.u.. 在腺嘌呤的四种加成产物中, ${ }^{5 \mathrm{OH}} \mathrm{A}-\mathrm{T}$ 的单电子轨道能量较高, 易失去电子, 其还原 性较强. 与之相反, ${ }^{2 \mathrm{OH}} \mathrm{A}-\mathrm{T}$ 和 ${ }^{4 \mathrm{OH}} \mathrm{A}-\mathrm{T}$ 单电子轨道能量 较低, 易得到电子, 具有较强的氧化性. 胸腺嘧啶的 四种加成产物中, $\mathrm{A}-\mathrm{T}^{5 \mathrm{OH}}$ 的单电子占据轨道能量较 高, 具有较强的还原性; $\mathrm{A}-\mathrm{T}^{2 \mathrm{OH}}$ 和 $\mathrm{A}-\mathrm{T}^{\mathrm{OOH}}$ 的单电子占 据轨道能量较低, 氧化性较强, 这与文献报道的结 果 $^{[1-6]}$ 一致.

图 5 给出了 8 种羟基化产物 SOMO 的分布情 况. 当碱基对中的腺嘌呤羟基化加成时, SOMO 主 要分布在腺嘌呤一侧; 但是当羟基对胸腺嘧啶加成 时, SOMO 的分布情况与加成位置有很大关系, 如

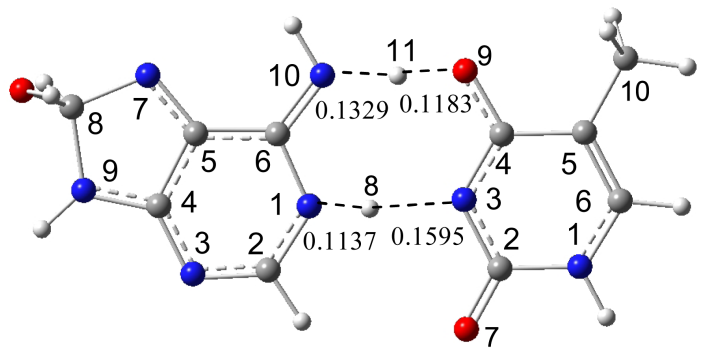

${ }^{8 \mathrm{OH}} \mathrm{A}-\mathrm{T}(\mathrm{Ts})$

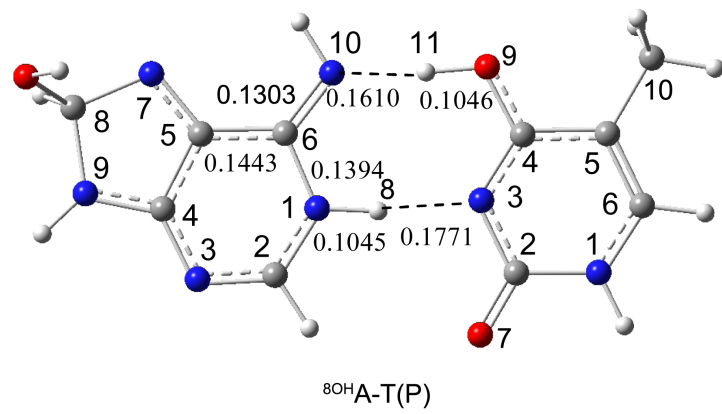

$\mathrm{A}-\mathrm{T}^{4 \mathrm{OH}}$ 和 $\mathrm{A}-\mathrm{T}^{5 \mathrm{OH}}$ 的 $\mathrm{SOMO}$ 主要分布在胸腺嘧啶一 侧, 而 $\mathrm{A}-\mathrm{T}^{2 \mathrm{OH}}$ 和 $\mathrm{A}-\mathrm{T}^{6 \mathrm{OH}}$ 的 $\mathrm{SOMO}$ 主要分布在腺嘌呤 一侧. ${ }^{2 \mathrm{OH}} \mathrm{A} 、{ }^{4 \mathrm{OH}} \mathrm{A} 、{ }^{5 \mathrm{OH}} \mathrm{A}$ 和 ${ }^{8 \mathrm{OH}} \mathrm{A}$ 的 SOMO 的能量分别 为 $-0.195 、-0.188 、-0.160$ 和 -0.181 a.u., 远高于胸 腺嘧啶的最高占据轨道(HOMO)的能量(-0.241 a.u.), 故当它们与胸腺嘧啶的 HOMO 作用时, SOMO 主要 分布在腺嘌呤一侧. $\mathrm{T}^{2 \mathrm{OH}} 、 \mathrm{~T}^{4 \mathrm{OH}} 、 \mathrm{~T}^{5 \mathrm{OH}}$ 及 $\mathrm{T}^{6 \mathrm{OH}}$ 的 $\mathrm{SOMO}$ 能量分别为 $-0.245 、-0.208 、-0.187$ 和 -0.239 a.u., 由于 $\mathrm{T}^{4 \mathrm{OH}}$ 和 $\mathrm{T}^{5 \mathrm{OH}}$ 的 $\mathrm{SOMO}$ 能量高于腺嘌呤的 HOMO 能量(-0.216 a.u.), 故 $\mathrm{A}-\mathrm{T}^{4 \mathrm{OH}}$ 和 $\mathrm{A}-\mathrm{T}^{5 \mathrm{OH}}$ 的 $\mathrm{SOMO}$ 主要 分布在胸腺嘧啶一侧, 而 $\mathrm{A}-\mathrm{T}^{2 \mathrm{OH}}$ 和 $\mathrm{A}-\mathrm{T}^{6 \mathrm{OH}}$ 的 $\mathrm{SOMO}$ 能量低于腺嘌呤的 HOMO 能量, 因此它们的 SOMO

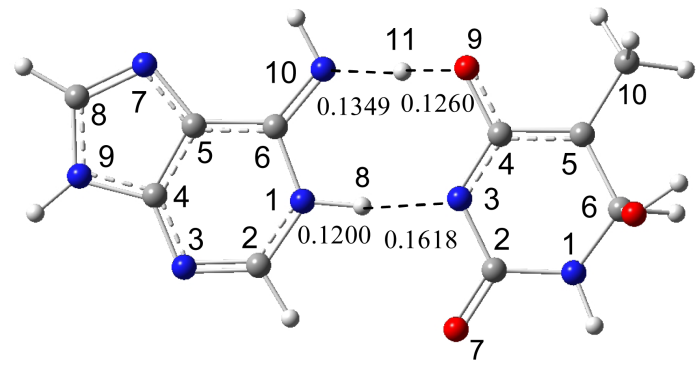

$\mathrm{A}-\mathrm{T}^{6 \mathrm{OH}}(\mathrm{Ts})$

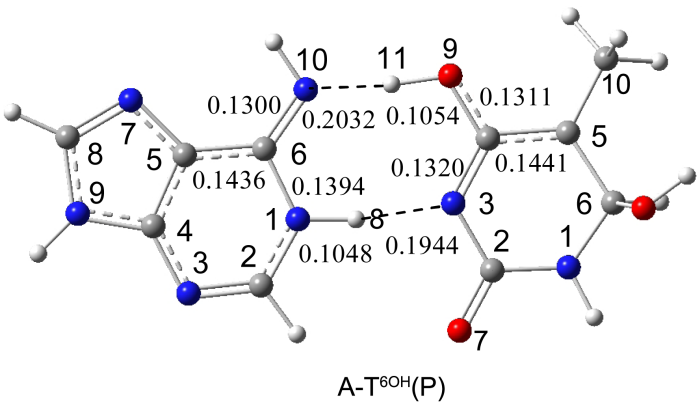

图 $6{ }^{80 \mathrm{H}} \mathrm{A}-\mathrm{T}$ 与 $\mathrm{A}-\mathrm{T}^{6 \mathrm{OH}}$ 质子转移过程所涉及的过渡态与产物的优化构型

Fig.6 Optimized structures of transition states and products of ${ }^{8 \mathrm{OH}} \mathrm{A}-\mathrm{T}$ and $\mathrm{A}-\mathrm{T}^{6 \mathrm{OH}}$ corresponding to the proton transfer reaction 
表 $3{ }^{80 \mathrm{H}} \mathrm{A}-\mathbf{T}$ 与 $\mathbf{A}-\mathbf{T}^{6 \mathrm{OH}}$ 质子转移过程所涉及的能垒和反应热

Table 3 Calculated activation barriers and reaction energies for the proton transfer reactions of ${ }^{80 \mathrm{H}} \mathrm{A}-\mathrm{T}$ and $\mathrm{A}-\mathrm{T}^{6 \mathrm{OH}}$

\begin{tabular}{cccc}
\hline System & $\Delta E_{\mathrm{f}} /\left(\mathrm{kJ} \cdot \mathrm{mol}^{-1}\right)$ & $\Delta E_{\mathrm{b}} /\left(\mathrm{kJ} \cdot \mathrm{mol}^{-1}\right)$ & $\Delta E /\left(\mathrm{kJ} \cdot \mathrm{mol}^{-1}\right)$ \\
\hline${ }^{8 \mathrm{OH}} \mathrm{A}-\mathrm{T}$ & $38.25(42.01)$ & $-10.09(0.90)$ & $48.34(41.11)$ \\
$\mathrm{A}-\mathrm{T}^{6 \mathrm{OOH}}$ & $47.69(50.27)$ & $-7.92(3.12)$ & $55.61(47.15)$ \\
\hline
\end{tabular}

$\Delta E_{\mathrm{f}}$ and $\Delta E_{\mathrm{b}}$ represent the forward and reverse activation barriers, respectively; $\Delta E$ is the reaction energy; the data in parentheses refer to the relative energies which do not include the zero-point energy.

主要分布在腺嘌呤一侧.

\section{2 质子转移的反应机理}

本文以能量较低的两种加成产物 ${ }^{8 \mathrm{OH}} \mathrm{A}-\mathrm{T}$ 和 $\mathrm{A}-\mathrm{T}^{6 \mathrm{OH}}$ 为例对羟基化 A-T 间的质子转移过程进行了 研究. 为便于比较, 在 $\mathrm{B} 3 \mathrm{LYP} / 6-31++\mathrm{G}(d, p) / / \mathrm{B} 3 \mathrm{LYP} /$ 6-31G $(d, p)$ 水平上对未羟基化 A-T 间的质子转移过 程进行了研究, 发现 A-T 间的质子转移为分步质 子转移, 其能垒分别为 62.31 和 $48.78 \mathrm{~kJ} \cdot \mathrm{mol}^{-1}$, 与 Kryachk等人的计算结果 ${ }^{[14]}$ 基本一致.

图 6 显示了羟基化碱基对之间质子转移的过渡 态及产物的构型. 表 3 中列出了 ${ }^{80 \mathrm{H}} \mathrm{A}-\mathrm{T}$ 与 $\mathrm{A}-\mathrm{T}^{6 \mathrm{OH}}$ 质 子转移过程所涉及的能垒和反应热. 我们对质子转 移过程中可能存在的离子型中间体进行了优化, 但 未能找到稳定的中间体, 即整个质子转移过程是协 同进行的. 本文对所得到的过渡态的结构进行了振 动频率计算, 并通过 IRC 路径分析予以确认.

计算表明羟基化 A-T 间的双质子转移发生在氢 键 N10(A) $\cdots \mathrm{O} 9(\mathrm{~T})$ 及 $\mathrm{N} 1(\mathrm{~A}) \cdots \mathrm{N} 3(\mathrm{~T})$ 之间, $\mathrm{H} 11$ 由腺嘌 呤转移到胸腺嘧啶的 $\mathrm{O} 9$ 上, 同时胸腺嘧啶的 $\mathrm{H} 8$ 向 腺嘌呤的 $\mathrm{N} 1$ 转移. 当 ${ }^{80 \mathrm{H}} \mathrm{A}-\mathrm{T}$ 和 $\mathrm{A}-\mathrm{T}^{6 \mathrm{6H}}$ 形成过渡态时 过渡态的构型更接近产物. 此结果符合 Hammond 假设 ${ }^{[26]}$, 即若质子转移的过程为吸热过程, 反应的过 渡态与产物的构型更相近.

表 3 表明, ${ }^{80 \mathrm{H}} \mathrm{A}-\mathrm{T}$ 和 $\mathrm{A}-\mathrm{T}^{60 \mathrm{H}}$ 发生协同双质子转移 时, 其势垒分别为 42.01 和 $50.27 \mathrm{~kJ} \cdot \mathrm{mol}^{-1}$, 均低于 $\mathrm{A}-\mathrm{T}$ 发生第一步质子转移(决速步骤)的能垒, 这说明 羟基对于腺嘌呤的 8 号位和胸腺嘧啶的 6 号位的羟 基加成有利于 A-T 间质子转移的进行. 另外, 两种加 成产物质子转移后的产物比质子转移前的能量高 48.34 和 $55.67 \mathrm{~kJ} \cdot \mathrm{mol}^{-1}$, 且与质子转移过渡态的能 量相差极小, 所以它们质子转移的产物极不稳定. ${ }^{8 \mathrm{OH}} \mathrm{A}-\mathrm{T}$ 和 $\mathrm{A}-\mathrm{T}^{6 \mathrm{OOH}}$ 发生质子转移的势垒之所以比 $\mathrm{A}-\mathrm{T}$ 发生第一步质子转移的势垒低, 与碱基对中碱基酸 碱性的变化有关. 表 4 中列出了 $\mathrm{A} / \mathrm{T}$ 及 ${ }^{8 \mathrm{OH}} \mathrm{A} / \mathrm{T}^{6 \mathrm{OH}}$ 中相
表 $4 \mathrm{~A} / \mathbf{T}$ 及 ${ }^{80 \mathrm{H}} \mathrm{A} / \mathbf{T}^{6 \mathrm{OH}}$ 中相关位置的去质子焓与质子亲和势

Table 4 Deprotonation enthalpies (DPE) and proton affinities (PA) for the relative positions of $\mathrm{A} / \mathrm{T}$ and ${ }^{8 \mathrm{OH}} \mathrm{A} / \mathbf{T}^{6 \mathrm{OH}}$

\begin{tabular}{cll}
\hline & $\mathrm{DPE}\left(\mathrm{kJ} \cdot \mathrm{mol}^{-1}\right)$ & $\mathrm{PA}\left(\mathrm{kJ} \cdot \mathrm{mol}^{-1}\right)$ \\
\hline $\mathrm{A}$ & $1466.43(\mathrm{~N} 10 \mathrm{H} 11)$ & $924.74(\mathrm{~N} 1)$ \\
${ }^{80 \mathrm{H}} \mathrm{A}$ & $1420.51(\mathrm{~N} 10 \mathrm{H} 11)$ & $911.00(\mathrm{~N} 1)$ \\
$\mathrm{T}$ & $1425.19(\mathrm{~N} 3 \mathrm{H} 8)$ & $838.94(\mathrm{O} 9)$ \\
$\mathrm{T}^{6 \mathrm{OH}}$ & $1407.78(\mathrm{~N} 3 \mathrm{H} 8)$ & $834.86(\mathrm{O} 9)$ \\
\hline
\end{tabular}

关位置的质子亲和势与去质子焓. 在 ${ }^{80 \mathrm{H}} \mathrm{A}$ 中,${ }^{80 \mathrm{H}} \mathrm{A}$ 端 $\mathrm{N} 10 \mathrm{H} 11$ 的去质子焓为 $1420.51 \mathrm{~kJ} \cdot \mathrm{mol}^{-1}$, 与碱基 A 相比减小了 $25.92 \mathrm{~kJ} \cdot \mathrm{mol}^{-1}$, 表明其酸性增强; $\mathrm{N} 1$ 位 的质子亲和势仅减小了 $13.74 \mathrm{~kJ} \cdot \mathrm{mol}^{-1}$, 这表明此位 置的碱性基本不变. 在 $\mathrm{T}^{6 \mathrm{OH}}$ 中, $\mathrm{N} 3$ 位的去质子焓与 $\mathrm{T}$ 相比减小 $17.41 \mathrm{~kJ} \cdot \mathrm{mol}^{-1}$, 酸性增强; 但 $\mathrm{O} 9$ 位置质子 亲和势仅降低 $4.08 \mathrm{~kJ} \cdot \mathrm{mol}^{-1}$, 碱性基本不变. 在对应 碱基不变的情况下, 羟基化碱基的碱性基本不变, 酸性增强, 质子更易解离, 故经羟基加成后 A-T 间的 质子转移势垒降低.

\section{3 结 论}

应用密度泛函理论在 B3LYP/6-31G $(d, p)$ 水平上 对羟基化碱基对 A-T 的结构进行了研究, 并在 B3LYP/6-31++ $\mathrm{G}(d, p)$ 水平上对相关结构进行单点能 计算. 经优化后共得到 8 种稳定的羟基化产物, 产物 能量的高低与加成发生前后的结构变化有密切关 系, 能量的相对顺序为 ${ }^{8 \mathrm{OH}} \mathrm{A}-\mathrm{T}<\mathrm{A}-\mathrm{T}^{6 \mathrm{OH}}<\mathrm{A}-\mathrm{T}^{5 \mathrm{OH}}<$ ${ }^{2 \mathrm{OH}} \mathrm{A}-\mathrm{T}<{ }^{4 \mathrm{OH}} \mathrm{A}-\mathrm{T}<{ }^{5 \mathrm{OH}} \mathrm{A}-\mathrm{T}<\mathrm{A}-\mathrm{T}^{2 \mathrm{OH}}<\mathrm{A}-\mathrm{T}^{4 \mathrm{OH}}$. 当羟基进 攻腺嘌呤时, 碱基对间的相互作用增加; 而当羟基 进攻胸腺嘧啶时, 其相互作用减弱. 在腺嘌呤端的 4 种加成产物中, ${ }^{20 \mathrm{H}} \mathrm{A}-\mathrm{T}$ 和 ${ }^{40 \mathrm{H}} \mathrm{A}-\mathrm{T}$ 具有较强的氧化性, ${ }^{5 \mathrm{OH}} \mathrm{A}-\mathrm{T}$ 具有较强的还原性; 在胸腺嘧啶端 4 种加成 产物中, $\mathrm{A}-\mathrm{T}^{5 \mathrm{OH}}$ 的还原性较强, $\mathrm{A}-\mathrm{T}^{2 \mathrm{OH}}$ 和 $\mathrm{A}-\mathrm{T}^{4 \mathrm{OH}}$ 的氧 化性较强; 经羟基化后 $\mathrm{A}-\mathrm{T}$ 的质子转移机理由分步 双质子转移变为协同双质子转移, 且其势垒低于 $\mathrm{A}-\mathrm{T}$ 发生质子转移的势垒.

\section{References}

1 Breen, A. P.; Murphy, J. A. Free Radical Bio. Med., 1995, 18: 1033

2 Burrows, C. J.; Muller, J. G. Chem. Rev., 1998, 98: 1109

3 Steenken, S.; Novais, H. M. J. Phys. Chem., 1987, 91: 426

4 Vieira, A. J. S. C.; Steenken, S. J. Am. Chem. Soc., 1990, 112: 6986

5 Steenken, S. Chem. Rev., 1989, 89: 503

6 Colson, A. O.; Sevilla, M. D. J. Phys. Chem., 1996, 100: 4420 
7 Lowdin, P. O. Adv. Quantum Chem., 1965, 2: 213

8 Piccirilli, J. A.; Krauch, T.; Moroney, S. E.; Benner, S. A. Nature, 1990, 343: 33

9 Benderskii, V. A.; Makarov, D. E.; Wight, C. A. Chemical dynamic at low temperature: advances in chemical physics. New York: Wiley \& Sons, 1994

10 Zhanpeisov, N. U.; Leszczynski, J. J. Phys. Chem. A, 1998, 102: 6167

11 Truhlar, D. G. Chem. Phys. Lett., 1998, 294: 45

12 Floribn, J.; Hrouda, V.; Pave1, H. J. Am. Chem. Soc., 1994, 116: 1457

13 Gorb, L.; Podolyan, Y.; Dziekonski, P.; Sokalski, A.; Lexzczynski, J. J. Am. Chem. Soc., 2004, 126: 10119

14 Kryachk, E. S.; Sabin, J. R. Int. J. Quantum Chem., 2003, 91: 695

15 Villani, G. Chem. Phys., 2005, 316: 1

16 Xie, H.; Xia, F.; Cao, Z. J. Phys. Chem. A, 2007, 111: 4384
17 Zhang, J. D.; Schaefee III, H. F. J. Chem. Theory Comput., 2007, 3: 115

18 Grand, A.; Morell, C.; Labet, V.; Cadet, J.; Eriksson, L. A. J. Phys. Chem. A, 2007, 111: 8968

19 Li, L.; Wang, H.; Niu, X. J.; Li, Z. H. Chem. J. Chin. Univ., 2009, 30: 1596 [李 澜, 王 竑, 牛晓娟, 李宗和. 高等学校化 学学报, 2009, 30: 1596]

20 Zhang, R. B.; Eriksson, L. A. J. Phys. Chem. B, 2007, 111: 6571

21 Hohenberg, P.; Kohn, W. Phys. Rev. B, 1964, 136: 864

22 Feller, D. J. Chem. Phys., 1990, 93: 579

23 Lee, C.; Yang, W.; Parr, R. G. Phys. Rev. B, 1988, 37: 785

24 Becke, A. D. J. Chem. Phys., 1993, 98: 5648

25 Frisch, M. J.; Trucks, G. W.; Schlegel, H. B.; et al. Gaussian 03. Revision B.03. Pittsburgh, PA: Gaussian Inc., 2003

26

\section{第九届东方胶化杯全国胶体化学研究生优秀成果奖评选通知}

为促进中国的胶体化学发展, 鼓励这一领域青年学生的进取精神, 2002 年起特设立全国胶体化学研究生奖学金, 以表彰优 秀的胶体化学专业研究生.

\section{申请资格与范围:}

全国范围内各大学、科研机构在读的胶体化学专业研究生(含博士生)均有资格申请. 申请人应在胶体与界面化学领域取得 较为突出的创新性结果. 发表一定数量的优秀科研论文 (含已接受). 申请人的所有成果必须是在研究生阶段进行或完成的. 在 职研究生不参与本奖学金评选. 对于积极参加全国胶体与界面专业委员会学术活动的科研小组, 将采取适度倾斜政策给予支 持.

\section{评选程序:}

1) 本人申请: 申请人需提交个人简历(含联系方式)、学术论文发表及已接受目录(含全部作者的正确顺序、期刊、年、卷(期)、 页), 科研工作自评, 论文工作概况. 已接受文章须具备期刊的接收函(含 Email). 并说明参加胶体与界面化学专业委员会的会议 情况.

2) 单位推荐: 申请人须有本单位两名副教授以上职称的老师(含导师)推荐. 各单位原则上最多推举两名.

3) 专家评审: 由全国胶体与界面化学专业委员会组织专家进行评审, 确定获奖名单.

\section{评选进程:}

2011 年 1 月 20 日前递交个人申请与推荐材料(含电子版、纸版), 经专家评审后于 2011 年 3 月正式公布并举行颁奖仪式.

联系人: 黄建滨 Tel: +86-10-62753557 Eamil: JBHuang@pku.edu.cn 邮寄地址: 北京大学化学与分子工程学院 邮政编码: 100871

本奖学金的设立获得了北京东方德菲胶体化学仪器有限公司大力支持与独家赞助. 本奖学金评选工作的最终解释权在全国胶体与界面化学专业委员会. 